\title{
Behavioral inhibition in female college students with schizotypal traits: An event-related potential study
}

\author{
Ji-Hyun Lee, Myung-Sun Kim* \\ Department of Psychology, Sungshin Women's University, Seoul, South Korea \\ Email: "kimms@,sungshin.ac.kr
}

Received 30 August 2012; revised 29 September 2012; accepted 8 October 2012

\begin{abstract}
This study investigated behavioral inhibition in female college students with psychometrically defined schizotypal traits using a Go/NoGo task and eventrelated potentials (ERPs). The schizotypal-trait (n = 15) and normal control $(n=15)$ groups were selected based on scores of the Schizotypal Personality Questionnaire (SPQ). The Go/NoGo task consisted of Go (requires response) and NoGo (requires no response) conditions. In terms of response time and accuracy rate for the Go/NoGo task, the two groups did not differ significantly. In terms of ERPs, the control group showed greater $\mathrm{N} 2$ amplitudes in response to NoGo (NoGo-N2) than to Go stimuli (Go-N2), whereas the schizotypal-trait group showed no significant difference in NoGo-N2 and Go-N2 amplitudes. In addition, the schizotypal-trait group showed reduced NoGo-N2 amplitudes at the frontal site compared to controls, and an association between SPQ scores and NoGo-N2 amplitudes measured at the frontal site. The two groups did not differ in $\mathrm{P} 3$ amplitudes. Since the $\mathrm{N} 2$ reflects the detection of response conflict and behavioral inhibition, the present results indicate that nonclinical individuals with schizotypal traits have difficulties in detecting response conflict and behavioral inhibition.
\end{abstract}

Keywords: Behavioral Inhibition; Event-Related Potentials; Go/NoGo Task; N2; P3; Schizotypal Trait

\section{INTRODUCTION}

Behavioral or response inhibition involves the control of overt behavior such as motor or impulsive responses irrelevant to goal-directed behavior [1]. The Go/NoGo task is widely used to measure behavioral inhibition, because it requires participants to respond to Go stimuli, but withhold responses to NoGo stimuli. Studies that have investigated behavioral inhibition in patients with

${ }^{*}$ Corresponding author. schizophrenia using the Go/NoGo task have reported that schizophrenia patients show significantly longer response times in response to Go stimuli [2] and more errors in response to NoGo stimuli than normal controls [3]. This indicates that patients with schizophrenia have deficits in behavioral inhibition.

Neuroimaging studies have provided neuroanatomical evidence underlying the lower performance on the Go/NoGo task in schizophrenia patients. For example, increased activations in the anterior cingulate cortex (ACC) and the dorsolateral prefrontal cortex are consistently observed during the performance of the NoGo condition of the Go/NoGo task in normal controls [4,5], whereas activations in these brain structures are significantly reduced in schizophrenia patients relative to controls $[6,7]$. In addition, structural abnormalities in the ACC and the dorsolateral prefrontal cortex have been reported in schizophrenia patients [8,9]. These findings suggest that the impaired performance on the Go/NoGo task observed in schizophrenia patients might be related to structural and functional abnormalities of frontal areas.

Event-related potentials (ERPs), the electrical brain activity time-locked to external events, have been widely used to investigate cognitive functions including behavioral inhibition due to the high temporal resolution associated with this technique. Several ERP studies have identified the electrophysiological indices of behavioral inhibition using the Go/NoGo task [3,10-12]. These ERP studies have demonstrated that two primary ERP components elicited in the NoGo condition, designated N2 and $\mathrm{P} 3$, are related to behavioral inhibition.

The amplitude of N2, a negative deflection observed over fronto-central sites 200 - $350 \mathrm{~ms}$ after stimulus onset, is greater in the NoGo condition (NoGo-N2) than in the Go condition (Go-N2) [13,14]. The NoGo-N2 reflects the inhibition of inappropriate responses and the detection or monitoring of conflict, and is also an index of efficiency of inhibition [15-17]. The ACC appears to be the source of NoGo-N2 [10,18,19], and neuroimaging studies have also documented increased activation of the 
ACC in the condition that requires response inhibition and detection or monitoring of conflict [20-22].

Another component related to response inhibition, P3, is a positive peak observed around $400-600 \mathrm{~ms}$ after stimulus onset. The P3 elicited in the NoGo condition (NoGo-P3) has a larger amplitude and a longer latency, and is more frontally localized than the P3 elicited in the Go condition (Go-P3) [10,23]. Although the functional significances of the NoGo-P3 are less well known than those of the NoGo-N2, the former may reflect behavioral inhibition and evaluation of behavioral plan [24].

Studies that have investigated behavioral inhibition in schizophrenia patients using the Go/NoGo task and ERPs have reported relatively consistent findings. For example, normal controls have shown larger N2 and P3 amplitudes in the NoGo condition than in the Go condition, whereas schizophrenia patients either have not shown significant differences in N2 and P3 amplitudes between NoGo and Go conditions or have shown reduced NoGo-N2 and NoGo-P3 amplitudes compared to normal controls [3, $25,26]$. These results indicate that schizophrenia patients have deficits in behavioral inhibition.

Given that schizophrenia is highly heterogeneous and that several variables including antipsychotic drug use and length of illness or hospitalization can affect cognitive functioning, patients with schizotypal personality disorder (SPD) and nonclinical individuals with schizotypal traits have been viewed as promising subjects for an endophenotypic approach to understanding schizophrenia [27]. Indeed, SPD and schizophrenia share common genetic [28], neuroimaging [29] and neuropsychological [30] abnormalities. Only a few studies have investigated behavioral inhibition in patients with SPD or in nonclinical individuals with schizotypal traits using the Go/NoGo task. For example, Gschwandtner et al. [31] investigated behavioral inhibition in individuals at highrisk for schizophrenia using the Go/NoGo task, and found that the high-risk group had significantly longer response times in the Go condition than did normal controls.

We investigated behavioral inhibition in nonclinical individuals with psychometrically defined schizotypal traits using the Go/NoGo task and ERPs. We were particularly interested in determining whether individuals with schizotypal traits show a deficit in behavioral inhibition, and if so, whether this deficit is reflected by ERP components such as N2 and P3.

\section{METHODS}

\subsection{Participants}

Thirty female college students were recruited from a pool of 400 students based on their scores on the Korean version of the Schizotypal Personality Questionnaire
(SPQ) [32,33]. The SPQ is a 74-item self-administered questionnaire with a "yes/no" response format. All items answered "yes" are scored as one, therefore, the total range of scores is $0-74$. The schizotypal-trait group $(\mathrm{n}=$ 15) was composed of those who obtained the highest $5 \%$ of scores on the SPQ [33] (score range: 36 -47), and the control group $(n=15)$ consisted of those who obtained average ( $\pm 1 \mathrm{SD}$ ) scores on the SPQ (score range: 16 - 22). The Structured Clinical Interview for DSM-IV-Non Patient (SCID-NP) [34] was administered to ensure that none of the participants had a history of psychiatric, medical, or neurologic disorders or of drug/alcohol abuse. All participants were right-handed, and none was taking medications at the time of testing. In addition, none of the participants had family history of psychiatric disorders. All participants provided written informed consent after receiving a complete description of the study, and they were paid for their participation. The study was approved by the Sungshin Women's University Institutional Bioethics Review Board.

\subsection{The Go/NoGo Task}

The computerized Go/NoGo task, which consists of two conditions (Go and NoGo conditions), was administered to measure behavioral inhibition. For half of the participants, red "O" and blue " $\mathrm{X}$ " were Go stimuli and blue "O" and red " $\mathrm{X}$ " were NoGo stimuli, whereas for the other half of the participants, blue " $\mathrm{O}$ " and red " $\mathrm{X}$ " were Go stimuli and red "O" and blue " $\mathrm{X}$ " were NoGo stimuli. A total of 600 stimuli (300 stimuli for each condition) were presented randomly in two blocks, and the participants were instructed to respond to the Go stimuli by pressing a button with the index finger of their right or left hand but not to respond to the NoGo stimuli. The hand position used for responses was counterbalanced across participants.

The stimuli were presented for $150 \mathrm{~ms}$ in foveal vision on a computer monitor using E-PRIME (Psychological Software Tools Inc., Sharpsburg, PA, USA), and each was subtended at a vertical visual angle of $3.5^{\circ}$ and a horizontal visual angle of $3.8^{\circ}$. A crosshair (+) was displayed on the screen for $500 \mathrm{~ms}$ as a fixation point, and the interstimulus interval alternated randomly between 1500 and $1800 \mathrm{~ms}$. Prior to the experimental session, a block of 24 practice trials was administered to ensure that the instructions were understood.

\subsection{Electrophysiological Recording Procedure}

Electroencephalographic activity (EEG) was recorded using a 64-channel Geodesic Sensor Net connected to a 64-channel, high-input impedance amplifier (Net Amp 300: Electrical Geodesics, Eugene, OR) in an electrically shielded and soundproofed experimental room. Each 
electrode was referenced to $\mathrm{Cz}$, and individual electrodes were adjusted until impedances were less than $50 \mathrm{k} \Omega$ [35]. Eye movements and blinks were monitored with electrodes placed near the outer cantus and beneath the left eye.

During the experiment, EEG activity was recorded continuously using a $0.1-100 \mathrm{~Hz}$ analog bandpass and a sampling rate of $250 \mathrm{~Hz}$. After the completion of data collection, the EEG was segmented into $1100 \mathrm{~ms}$ epochs (including a $200 \mathrm{~ms}$ pre-stimulus baseline) with respect to the event markers. The epochs that were contaminated by artifacts such as eye blinks and eye movements were rejected before averaging (the threshold for artifact rejection was $\pm 70 \mu \mathrm{V}$ ). Data were then averaged for each subject and each condition (Go and NoGo conditions). An average-reference transformation was used to minimize the effects of reference-site activity [36]. ERPs were baseline-corrected with respect to the $200 \mathrm{~ms}$ pre-stimulus recording interval, and were digitally low-pass filtered at $30 \mathrm{~Hz}$. Only those EEGs associated with correct responses were subjected to statistical analyses.

\subsection{Statistical Analysis}

Based on visual inspection of grand average and individual ERP waveforms, two ERP components (N2 and P3) and three regions of interest (ROI) (i.e. frontal [Fz, $\mathrm{F} 3, \mathrm{~F} 4]$, central $[\mathrm{Cz}, \mathrm{C} 3, \mathrm{C} 4]$ and parietal $[\mathrm{Pz}, \mathrm{P} 3, \mathrm{P} 4]$ areas) were selected. $\mathrm{N} 2$ was defined as the most negative peak observed $200-350 \mathrm{~ms}$ after stimulus-onset, while P3 amplitude was defined as the mean amplitude observed 400 - $600 \mathrm{~ms}$ after stimulus-onset because we could not observe a distinctive positive peak in this time window. The N2 amplitudes and latencies and P3 amplitudes were separately analyzed with a repeated measure, mixed-design analysis of variance (ANOVA) with stimulus condition (Go and NoGo conditions) and ROI (frontal, central, and parietal areas) as within-subject factors and group (schizotypal-trait and control groups) as the between-subject factor. Greenhouse-Geisser corrections for sphericity violations were employed when appropriate, and the corrected $\mathrm{p}$ values are reported. Variables showing significant main effects were further analyzed using a paired t-test or a one-way ANOVA.

The response time in the Go condition was analyzed with a one-way ANOVA, and the accuracy was subjected to a repeated measure, mixed-design ANOVA with stimulus condition as the within-subject factor and group as the between-subject factor. The demographic characteristics of the participants included in the schizotypal-trait and control groups were compared using a one-way ANOVA. We employed a two-tailed Pearson's correlation to determine the relationships between the $\mathrm{N} 2 / \mathrm{P} 3$ and schizotypal symptoms as evaluated by the
SPQ, and between N2/P3 and behavioral performance on the $\mathrm{Go} / \mathrm{NoGo}$ task.

\section{RESULTS}

\subsection{Demographic Characteristics}

The schizotypal-trait and control groups did not differ significantly in age $\left(F_{1,28}=0.48, n s\right)$ or educational level $\left(F_{1,28}=0.29, n s\right)$, and their mean ages were $20.60(\mathrm{SD}=$ $1.68)$ and $20.13(\mathrm{SD}=2.00)$ years, respectively. In addition, the mean years of education completed by the schizotypal-trait and control groups were 14.13 (SD = $1.06)$ and $14.33(\mathrm{SD}=0.98)$ years, respectively. However, the SPQ scores of the two groups differed significantly $\left(F_{1,28}=327.67, p<0.0001\right)$ in that the schizotypal-trait group obtained significantly higher SPQ scores (mean $=40.27, \mathrm{SD}=4.18)$ than the control group $($ mean $=18.80, \mathrm{SD}=1.90)$.

\subsection{Behavioral Results of the Go/NoGo Task}

Statistical analysis of response times during the Go condition showed that schizotypal-trait and control groups did not differ significantly $\left(F_{1,28}=1.59, n s\right)$, although the schizotypal-trait group showed longer response times than the control group $(519.10 \mathrm{~ms}$ vs. $486.11 \mathrm{~ms})$. In terms of accuracy, we found a main effect of stimulus condition $\left(F_{1,28}=4.69, p<0.05\right)$. The NoGo stimuli elicited significantly more errors than did the Go stimuli. The mean accuracy rates of NoGo and Go conditions were $95.67 \%(\mathrm{SD}=0.76)$ and $97.87 \%(\mathrm{SD}=0.65)$, respectively. However, the accuracies of the two groups were not significantly different $\left(F_{1,28}=0.38, n s\right)$. The mean accuracy rates of the schizotypal-trait and control groups were $96.47 \%(\mathrm{SD}=0.69)$ and $97.07 \%(\mathrm{SD}=$ 0.70 ), respectively.

\subsection{ERP Results on the Go/NoGo Task}

The grand average ERPs elicited at $\mathrm{Fz}$ in response to Go and NoGo stimuli are depicted in Figure 1(a) for both groups. In terms of N2, the control group showed larger amplitudes in response to NoGo stimuli than to Go stimuli, whereas differences in N2 amplitudes between Go and NoGo stimuli were not observed in the schizotypal-trait group. Both groups showed larger P3 amplitudes in response to NoGo stimuli than to Go stimuli at Fz. The topographical distributions of different amplitudes (NoGo minus Go conditions) of N2 and P3 are presented in Figure 1(b). The NoGo condition elicited larger N2 and $\mathrm{P} 3$ amplitudes than did the Go condition in frontocentral and frontal areas, respectively.

The main effects of stimulus condition $\left(F_{1,28}=28.59\right.$, $p<0.0001)$ and ROI $\left(F_{2,56}=4.00, p<0.05\right)$ were observed for N2 amplitudes. The NoGo stimuli elicited 
larger N2 amplitudes than did Go stimuli and the amplitude measured at the frontal site was the largest, while the amplitude measured at the parietal site was the smallest. In addition, an interaction effect of group by stimulus condition $\left(F_{1,28}=10.19, p<0.001\right)$ was identified. In the control group, the NoGo stimuli elicited larger N2 amplitudes than did Go stimuli $\left(F_{1,28}=4.58, p<\right.$ $0.05)$, whereas no significant difference was observed between NoGo and Go stimuli in the schizotypal-trait group $\left(F_{1,28}=0.33, n s\right)$. The schizotypal-trait group exhibited significantly smaller N2 amplitudes at the frontal site in the NoGo condition compared to the control group $\left(F_{1,28}=7.34, p<0.01\right)$. We also observed an interaction effect of ROI by stimulus condition $\left(F_{2,56}=10.09\right.$. $p<$ $0.0001)$. The NoGo stimuli elicited larger N2 amplitudes

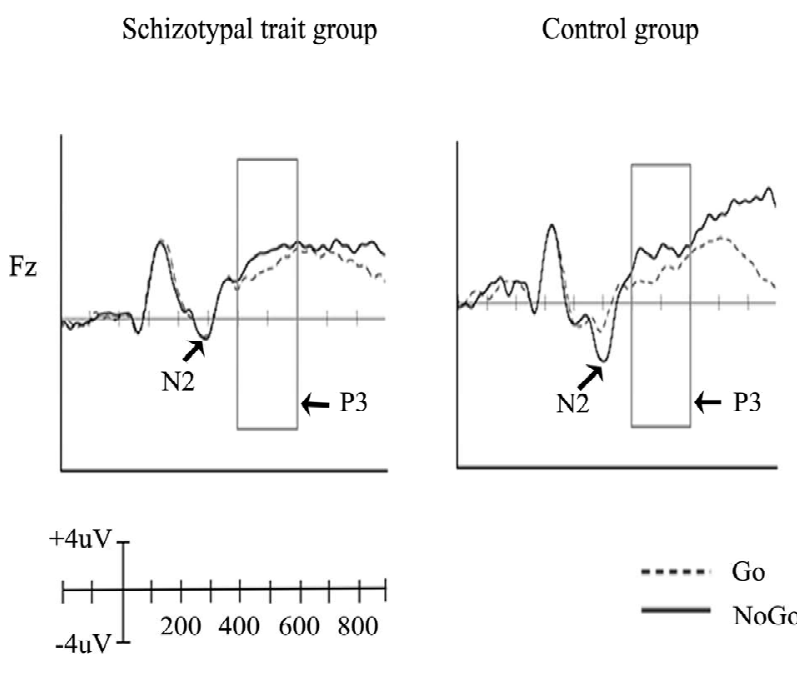

(a) than did Go stimuli at frontal $\left(t_{29}=5.25, p<0.0001\right)$ and central sites $\left(t_{29}=4.56, p<0.0001\right)$ but not at the parietal site $\left(t_{29}=0.75, n s\right)$. Only a main effect of ROI $\left(F_{2,56}=\right.$ $112.90, p<0.0001)$ was observed for N2 latency with the longest latency at the frontal site. The mean amplitudes and latencies of $\mathrm{N} 2$ obtained at each condition and ROI in the two groups are presented in Table 1.

The statistical analysis of P3 amplitudes showed a main effect of ROI $\left(F_{2,56}=14.66, p<0.0001\right)$ and an interaction effect of ROI by stimulus condition $\left(F_{2,56}=\right.$ $31.73, p<0.0001)$. The largest P3 amplitude was observed at the central site and the smallest amplitude was observed at the frontal site. The NoGo stimuli elicited greater P3 amplitudes than did Go stimuli at the frontal site $\left(t_{29}=-2.39, p<0.05\right)$, whereas Go stimuli elicited

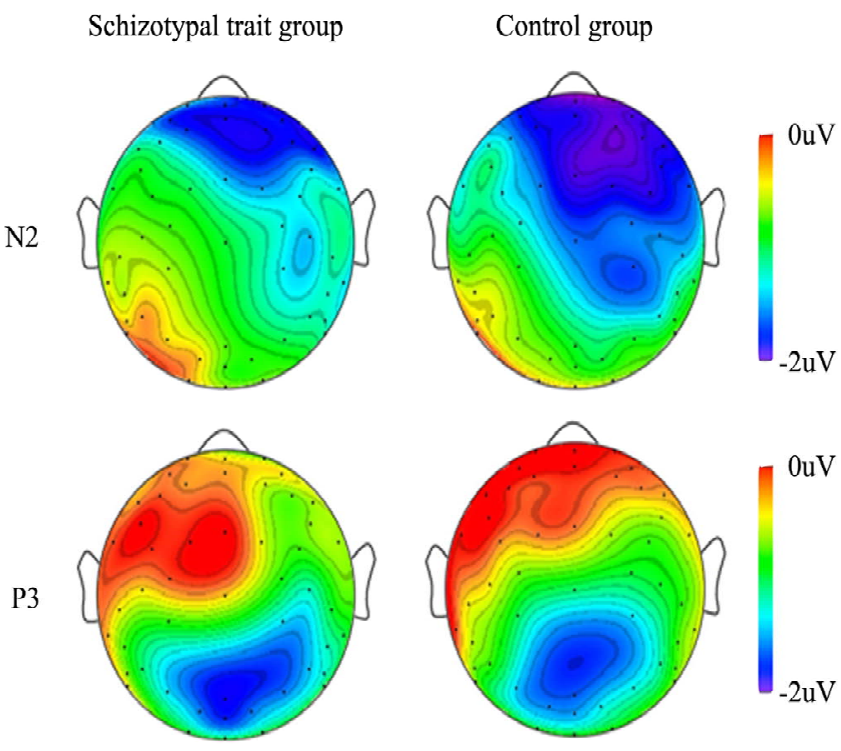

(b)

Figure 1. Grand average ERPs elicited at Fz in response to Go and NoGo stimuli (a), and topographical distributions of different amplitudes (NoGo minus Go conditions) of N2 and P3 (b).

Table 1. The mean amplitudes and latencies of N2 obtained at each condition and ROI in schizotypal-trait and control groups.

\begin{tabular}{|c|c|c|c|c|}
\hline & \multicolumn{2}{|c|}{ Schizotypal-trait group $(\mathrm{n}=15)$} & \multicolumn{2}{|c|}{ Control group $(n=15)$} \\
\hline & Go & NoGo & Go & NoGo \\
\hline \multicolumn{5}{|c|}{ Amplitude (uV) } \\
\hline Frontal & $-1.59(1.29)$ & $-1.93(1.39)$ & $-2.79(2.37)$ & $-3.77(2.22)$ \\
\hline Central & $-1.16(1.32)$ & $-1.63(1.25)$ & $-1.06(1.52)$ & $-2.08(1.54)$ \\
\hline Parietal & $-2.03(1.52)$ & $-1.82(1.58)$ & $-1.18(1.65)$ & $-1.59(1.28)$ \\
\hline \multicolumn{5}{|c|}{ Latency (ms) } \\
\hline Frontal & $311.02(22.86)$ & $313.76(24.03)$ & $285.84(37.07)$ & $285.98(37.68)$ \\
\hline Central & $283.00(33.27)$ & $285.44(34.08)$ & $275.02(44.58)$ & $270.40(45.17)$ \\
\hline Parietal & 275.07 (12.94) & 276.04 (11.85) & $270.29(20.70)$ & $273.11(25.00)$ \\
\hline
\end{tabular}

$\mathrm{SD}$ in parenthesis. 
larger amplitudes than did NoGo stimuli at central $\left(t_{29}=\right.$ $5.18, p<0.0001)$ and parietal sites $\left(t_{29}=8.59, p<0.0001\right)$ in both groups. The mean amplitudes of $\mathrm{P} 3$ obtained under each condition and ROI in schizotypal-trait and control groups are presented in Table 2.

\subsection{Correlations between N2/P3 and SPQ, and Behavioral Performance}

We found a significant correlation between SPQ total score and NoGo-N2 amplitude at the frontal site $(r=$ $-0.53, p<0.05$ ) in the schizotypal-trait group. The relationships between N2/P3 amplitudes and behavioral performance were not significant.

\section{DISCUSSION}

We used the Go/NoGo task and ERPs to investigate whether nonclinical individuals with psychometrically defined schizotypal traits have a deficit in behavioral inhibition. The results of the Go/NoGo task showed that both schizotypal-trait and control groups showed more errors in response to NoGo stimuli than to Go stimuli. The schizotypal-trait group showed longer response times in response to Go stimuli than did the control group, however, the difference in response times between the two groups was not statistically significant. These behavioral results are not consistent with those of previous studies, which observed that schizophrenia patients showed longer response times in response to Go stimuli $[2,37,38]$ and more errors in response to NoGo stimuli than did normal controls $[3,39,40]$. Different ratios for the Go and NoGo stimuli employed in previous studies and in our study may contribute to the inconsistent behavioral results. In our study, we used a 50:50 ratio for the Go and NoGo stimuli because the probabilities of stimulus-presentation are known to affect the amplitudes of ERP components, i.e. P3 [41]. In contrast, previous studies presented Go stimuli more frequently than NoGo stimuli (e.g. a 88:12 ratio) [40] to enhance the difficulty of inhibiting the response to the NoGo stimuli [14]. In addition, inconsistent findings may be attributable to differences among study participants. For example, in previous studies, first-episode patients with schizophre- nia did not show significantly more errors in response to NoGo stimuli than did normal controls [37], whereas chronic patients showed significantly more errors than did normal controls [40,42]. Therefore, the absence of behavioral differences in the Go/NoGo task between schizotypal-trait and control groups seems to be related to methodological differences such as ratios for Go and NoGo stimuli and the characteristics of the participants in the studies.

The control group showed larger N2 amplitudes in response to NoGo stimuli relative to Go stimuli, whereas no significant difference in N2 amplitudes between Go and NoGo stimuli was observed in the schizotypal-trait group. In addition, the schizotypal-trait group showed significantly reduced NoGo-N2 amplitudes compared to the control group, and NoGo-N2 amplitude elicited at the frontal site was significantly associated with schizotypal symptoms. These results are consistent with those of previous studies that observed reduced NoGo-N2 amplitude or no significant difference in N2 amplitudes between Go and NoGo stimuli in schizophrenia patients $[3,25,26]$. The $\mathrm{N} 2$ reflects inhibition of inappropriate behavior, detections of response conflict or ability to monitor response-decision, and is an index of efficient response inhibition [15-17], because relative to the Go condition the N2 amplitude is larger in the NoGo condition, which requires response inhibition and detection of response conflict $[19,24]$. Therefore, the present results indicate that individuals with schizotypal traits have difficulty in detecting response conflict and response inhibition.

The anterior cingulate cortex (ACC), which is involved in behavioral inhibition, conflict detection, and monitoring of response decisions, has been reported to be the source of the N2 $[6,18,43]$. Increased activation of the ACC has been observed during the performance of the NoGo task relative to the Go task in normal controls $[10,44]$, and schizophrenia patients show decreased activation of the ACC than normal controls during the performance of the NoGo task [45,46]. In addition, some studies have suggested that reduced NoGo-N2 amplitudes or no significant difference between NoGo-N2 and Go-N2 amplitudes in schizophrenia patients may result

Table 2. The mean P3 amplitudes obtained at each condition and ROI in schizotypal-trait and control groups.

\begin{tabular}{ccccc}
\hline & \multicolumn{2}{c}{ Schizotypal-trait group $(\mathrm{n}=15)$} & \multicolumn{2}{c}{ Control group $(\mathrm{n}=15)$} \\
\cline { 2 - 5 } & Go & NoGo & Go & NoGo \\
\hline Amplitude $(\mathrm{uV})$ & & & & $2.01(1.83)$ \\
Frontal & $3.06(1.51)$ & $3.15(1.67)$ & $5.24(1.65)$ & $4.03(1.58)$ \\
Central & $4.51(2.06)$ & $3.74(1.75)$ & $3.94(1.54)$ & $2.33(1.09)$ \\
Parietal & $3.90(1.66)$ & $2.11(1.34)$ & \\
\hline
\end{tabular}

$\mathrm{SD}$ in parenthesis. 
from decreased activation of the ACC, which leads to impairment of response inhibition and conflict detection $[20,25,45]$. Furthermore, structural and functional abnormalities of the ACC are observed in patients with schizophrenia [8] and SPD [47]. Therefore, the present results indicate that nonclinical individuals with schizotypal traits have difficulty with behavioral inhibition, which might be associated with ACC dysfunction. A previous neuroimaging study reported reduced activation of the ACC in first-episode schizophrenia patients than in normal controls during the performance of the Go/NoGo task, although the behavioral performances between patients and normal controls did not differ [7]. Taken together, these results and those of the present study indicate that dysfunction of the neural network involved in inhibition occurs even before the emergence of behavioral deficits.

Both groups exhibited greater P3 amplitudes in the NoGo condition than in the Go condition at the frontal site, consistent with previous findings [10,23]. However, contrary to previous studies that observed significantly reduced NoGo-P3 amplitudes in schizophrenia patients relative to normal controls [3,39], we did not identify any significant differences in the amplitudes of NoGo-P3 between individuals with schizotypal traits and controls. There is currently no consensus regarding the functional significance of NoGo-P3 in relation to response inhibition. For example, Falkenstein et al. [11] insisted that NoGo-P3 might actually reflect the reset or closure of a preceding inhibition process, because NoGo-P3 latency is too late to be an inhibition mechanism. In addition, some studies have observed no differences in the amplitude of NoGo-P3 in situations in which inhibition was either likely or unlikely [48]. Considering these suggestions, our findings suggest that NoGo-N2 is a better index of response inhibition than NoGo-P3.

Our study has several limitations that should be addressed in future studies. First, the inclusion of only a small number of female participants limits the generalizability of the findings. Second, findings of structural abnormalities of the ACC in patients with SPD [47] suggest that future studies should use both structural-functional brain imaging techniques and ERPs to enhance understanding of the neurophysiological mechanisms underlying the impairment of behavioral inhibition experienced by those with schizophrenia and SPD.

\section{CONCLUSION}

Schizotypal-trait and control groups did not differ in their behavioral performances of the Go/NoGo task. However, with regards to ERPs, the controls showed significantly larger N2 amplitudes in the NoGo condition relative to the Go condition, whereas nonclinical individuals with schizotypal traits displayed no difference between NoGo-
N2 and Go-N2 amplitudes and had reduced NoGo-N2 amplitudes at the frontal site compared to controls. Also NoGo-N2 amplitudes at the frontal site were negatively associated with SPQ scores in the schizotypal-trait group. These findings suggest that individuals with schizotypal traits have difficulty with behavioral inhibition, and dysfunction of the neural network involved in inhibition occurs even before the emergence of behavioral deficits.

\section{ACKNOWLEDGEMENTS}

This work was supported by the Sungshin Women's University Research Grant of 2011.

\section{REFERENCES}

[1] Harnishfeger, K.K. (1995) The development of cognitive inhibition: Theories, definitions and research evidence. In: Dempster, F.N. and Brainerd, C.J. Eds., New perspectives on interference and inhibition in cognition. Academic Press, San Diego, pp. 176-199

[2] Woolard, A.A., Kose, S., Woodward, N.D., Verbruggen, F., Logan, G.D. and Heckers, S. (2010) Intact associative learning in patients with schizophrenia: Evidence from a Go/NoGo paradigm. Schizophrenia Research, 122, 131135. doi:10.1016/j.schres.2010.02.1057

[3] Weisbrod, M., Kiefer, M., Marzinzik, F. and Spitzer, M. (2000) Executive control is disturbed in schizophrenia: Evidence from event-related potentials in a $\mathrm{Go} / \mathrm{NoGo}$ task. Society of Biological Psychiatry, 47, 51-60. doi:10.1016/S0006-3223(99)00218-8

[4] Garavan, H., Ross, T.J., Murphy, K., Roche, R.A.P. and Stein, E.A. (2002) Dissociable executive functions in the dynamic control of behavior: Inhibition, error detection, and correction. NeuroImage, 17, 1820-1829. doi:10.1006/nimg.2002.1326

[5] Liddle, P.F., Kiehl, K.A. and Smith, A.M. (2001) Eventrelated fMRI study of response inhibition. Human Brain Mapping, 12, 100-109.

doi:10.1002/1097-0193(200102)12:2<100::AID-HBM10 07>3.0.CO;2-6

[6] Kerns, J.G., Cohen, J.D., MacDonald, A.W., Johnson, M.K., Stenger, V.A., Aizenstein, H. and Carter, C.S. (2005) Decreased conflict- and error-related activity in the anterior cingulate cortex in subjects with schizophrenia. American Journal of Psychiatry, 162, 1833-1839. doi:10.1176/appi.ajp.162.10.1833

[7] Rubia, K., Russell, T., Bullmore, E.T., Soni, W., Brammer, M.J., Simmons, A., Taylor, E., Andrew, C., Giampietro, V. and Sharma, T. (2001) An fMRI study of reduced left prefrontal activation in schizophrenia during normal inhibitory function. Schizophrenia Research, 52, 47-55. doi:10.1016/S0920-9964(00)00173-0

[8] Albanese, A.M., Merlo, A.B., Mascitti, T.A., Tornese, E.B., Gomez, E.E., Konopka, V. and Albanese, E.F. (1995) Inversion of the hemispheric laterality of the anterior cingulate gyrus in schizophrenics. Biological Psychiatry, 38, 13-21. doi:10.1016/0006-3223(94)00257-4 
[9] Pantelis, C., Yucel, M., Wood, S.J., McGorry, P. and Velakoulis, P. (2001) The timing and functional consequences of structural brain abnormalities in schizophrenia. Neuroscience News, 4, 36-46.

[10] Bokura, H., Yamaguchi, S. and Kobayashi, S. (2001) Electrophysiological correlates for response inhibition in a Go/NoGo task. Clinical Neurophysiology, 112, 22242232. doi:10.1016/S1388-2457(01)00691-5

[11] Falkenstein, M., Hoormann, J. and Hohnsbein, J. (1999) ERP components in $\mathrm{Go} / \mathrm{NoGo}$ task and their relation to inhibition. Acta Psychologica, 101, 267-291. doi:10.1016/S0001-6918(99)00008-6

[12] Kim, M.S., Kim, Y.Y., Yoo, S.Y. and Kwon, J.S. (2007) Electrophysiological correlates of behavioral response inhibition in patients with obsessive-compulsive disorder. Depression and Anxiety, 24, 22-31. doi:10.1002/da.20195

[13] Fallgatter, A.J. and Strik, W.K. (1999) The NoGo-anteriorization as a neurophysiological standard-index for cognitive response control. International Journal of Psychophysiology, 32, 233-238. doi:10.1016/S0167-8760(99)00018-5

[14] Jodo, E. and Kayama, Y. (1992) Relation of a negative ERP component to response inhibition in a Go/NoGo task. Electroencephalography and Clinical Neurophysiology, 82, 477-482. doi:10.1016/0013-4694(92)90054-L

[15] Donkers, F.C.L. and van Boxtel, G.J.M. (2004) The N2 in Go/NoGo task reflects conflict monitoring not response inhibition. Brain and Cognition, 56, 165-176. doi:10.1016/j.bandc.2004.04.005

[16] Ruchsow, M., Groen, G., Kiefer, M., Beschoner, P., Hermle, L., Ebert, D. and Falkenstein, M. (2008) Electrophysiological evidence for reduced inhibitory control in depressed patients in partial remission: A Go/NoGo study. International Journal of Psychophysiology, 68, 209-218. doi:10.1016/i.ijpsycho.2008.01.010

[17] Yeung, N., Cohen, J.D. and Botvinick, M.M. (2004) The neural basis of error detection: conflict monitoring and the error-related negativity. Psychological Review, 111, 931-959. doi:10.1037/0033-295X.111.4.931

[18] Bekker, E.M., Kenemans, J.L. and Verbaten, M.N. (2005) Source analysis of the N2 in an cued Go/NoGo task. Cognitive Brain Research, 22, 221-231. doi:10.1016/j.cogbrainres.2004.08.011

[19] Nieuwenhuis, S., Yeung, N., van den Wildenberg, W. and Ridderinkhof, K.R. (2003) Electrophysiological correlates of anterior cingulate function in a Go/NoGo task: Effects of response conflict and trial type frequency. Cognitive, Affective, \& Behavioral Neuroscience, 3, 1726. doi:10.3758/CABN.3.1.17

[20] Carter, C.S., Braver, T.S., Barch, D.M., Botvinick, M.M., Noll, D. and Cohen, J.D. (1998) Anterior cingulate cortex, error detection, and the online monitoring of performance. Science, 280, 747-749. doi:10.1126/science.280.5364.747

[21] Lau, H., Rogers, R.D. and Passingham, R.E. (2006) Dissociating response selection and conflict in the medial frontal surface. NeuroImage, 29, 446-451. doi:10.1016/j.neuroimage.2005.07.050

[22] Walton, M.E., Devli, J.T. and Rushworth, M.F.S. (2004) Interactions between decision making and performance monitoring with prefrontal cortex. Nature Neuroscience, 7, 1259-1265. doi:10.1038/nn1339

[23] Strik, W.K., Fallgatter, A.J., Brandies, D. and PascualMarqui, R.D. (1998) Three-dimensional tomography of event-related potentials during response inhibition: Evidence for phasic frontal lobe activation. Electroencephalography and Clinical Neurophysiology, 108, 406-413. doi:10.1016/S0168-5597(98)00021-5

[24] Bruin, K.J. and Wijers, A.A. (2002). Inhibition, response mode, and stimulus probability: A comparative event-related potential study. Clinical Neurophysiology, 113, 1172-1182. doi:10.1016/S1388-2457(02)00141-4

[25] Kiehl, K.A., Smith, A.M., Hare, R.D. and Liddle, P.F. (2000) An event-related potential investigation of response inhibition in schizophrenia and psychopathy. Society of Biological Psychiatry, 48, 210-221. doi:10.1016/S0006-3223(00)00834-9

[26] Salisbury, D.F., O’Donnell, B.F., McCarley, R.W., Shenton, M.E. and Benavage, A. (1994) The N2 eventrelated potential reflects attention deficit in schizophrenia. Biological Psychology, 39, 1-13. doi:10.1016/0301-0511(94)90053-1

[27] Siever, L.J. and Davis, K.L. (2004) The pathophysiology of schizophrenia disorders: perspectives from the spectrum. American Journal of Psychiatry, 161, 398-413. doi:10.1176/appi.ajp.161.3.398

[28] Lin, H.F., Liu, Y.L., Liu, C.M., Hung, S.I., Hwu, H.G. and Chen, W.J. (2005) Neuregulin gene and variations in perceptual aberration of schizotypal personality in adolescents. Psychological Medicine, 35, 1589-1598. doi:10.1017/S0033291705005957

[29] Moorhead, T.W.J., Stanfield, A., Spencer, M., Hall, J., McIntosh, A., Qwnes, D.C., Lawrie, S. and Johnstone, E. (2009) Progressive temporal lobe gray matter loss in adolescents with schizotypal traits and mild intellectual impairment. Psychiatry Research: Neuroimaging, 174, 105-109. doi:10.1016/j.pscychresns.2009.04.003

[30] Noguchi, H., Hori, H. and Kunigi, H. (2008) Schizotypal traits and cognitive function in healthy adults. Psychiatry Research, 161, 162-169. doi:10.1016/i.psychres.2007.07.023

[31] Gschwandtner, U., Aston, J., Borgwardt, S., Drewe, M., Feinendegen, C., Lacher, D., Lanzarone, A., Stiegliz, R.D. and Riecher-Rőssler, A. (2003) Neuropsychological and neurophysiological findings in individuals suspected to be at risk for schizophrenia: preliminary results from the Basel early detection of psychosis study-Früherkennung von Psychosen (FEPSY). Acta Psychiatrica Scandinavica, 108, 152-155. doi:10.1034/j.1600-0447.2003.00157.x

[32] Moon, H.O., Yang, I.H., Lee, H.P., Kim, M.E. and Ham, W. (1997) The preliminary study on the validation of Schizotypal Personality Questionnaire-Korean version. Journal of Korean Neuropsychiatry Association, 36, 329343.

[33] Raine, A. (1991) The SPQ: A scale for the assessment of 
schizotypal personality based on DSM-III-R criteria. Schizophrenia Bulletin, 17, 555-564.

[34] First, M.B., Spitzer, R.L., Gibbson, M. and Williams, J.B.W. (1996) Structured clinical interview for DSM-IV Axis I disorder. New York State Psychiatric Institute, New York.

[35] Tucker, D.M. (1993) Spatial sampling of head electrical fields: the geodesic sensor net. Electroencephalography and Clinical Neurophysiology, 87, 154-163. doi:10.1016/0013-4694(93)90121-B

[36] Dien, J. (1998) Issues in the application of the average reference: Review, critiques, and recommendations. Behavior Research Methods, Instruments, \& Computers, 30, 34-43. doi:10.3758/BF03209414

[37] Begre, S., Kleinlogel, H., Kiefer, C., Strik, W., Dierks, T. and Federspiel, A. (2008) White matter anisotropy related to electrophysiology of first episode schizophrenia during NoGo inhibition. Neurobiology of Disease, 30, 270-280.

[38] Kaladjian, A., Jeanningros, R., Azorin, J.M., Grimault, S., Anton, J.L. and Mazzola-Pomietto, P. (2007) Blunted activation in right ventrolateral prefrontal cortex during motor response inhibition in schizophrenia. Schizophrenia Research, 97, 184-193. doi:10.1016/j.schres.2007.07.033

[39] Pallanti, S., Castrllini, G., Chamberlain, S.R., Quercioli, L., Zaccara, G. and Finegerg, N.A. (2009) Cognitive event-related potentials differentiate schizophrenia with obsessive-compulsive disorder (schizo-OCD) from OCD and schizophrenia without OC symptoms. Psychiatry Research, 170, 52-60. doi:10.1016/j.psychres.2008.11.002

[40] Ford, J.M., Gray, M., Whitfield, S.L., Turken, A.U., Gloverm G., Faustman, W.O. and Mathalon, D.H. (2004) Acquiring and inhibiting prepotent response in schizophrenia. Archives of General Psychiatry, 61, 119-129. doi:10.1001/archpsyc.61.2.119
[41] Polich, J. (2007) Updating P300: An integrative theory of P3a and P3b. Clinical Neurophysiology, 118, 2128-2148. doi:10.1016/j.clinph.2007.04.019

[42] Cornblatt, B.A. and Keilp, J.G. (1994) Impaired attention, genetics, and the pathophysiology of schizophrenia. Schizophrenia Bulletin, 20, 31-46.

[43] Carriero, L., Zalla, T., Budai, R. and Battaglini, P.P. (2007) Inhibition of wrong responses and conflict resolution: An electroencephalogram study. Neuroreport, 18, 793-796. doi:10.1097/WNR.0b013e3280c1e330

[44] Thomas, S.J., Gonsalvez, C.J. and Johnstone, S.J. (2009) Sequence effects in the Go/NoGo task: Inhibition and facilitation. International Journal of Psychophysiology, 74, 209-219. doi:10.1016/j.ijpsycho.2009.09.002

[45] Fallgatter, A.J., Bartsch, A.J., Zielasek, J. and Herrmann, M.J. (2003) Brain electrical dysfunction of the anterior cingulate in schizophrenic patients. Psychiatry Research: Neuroimaging, 124, 37-48. doi:10.1016/S0925-4927(03)00072-6

[46] Arce, E., Leland, D.S., Miller, D.A., Simmons, A.N., Winternheimer, K.C. and Paulus, M.P. (2006) Individuals with schizophrenia present hypo-and hyperactivation during implicit cueing in an inhibitory task. Neuroimage, 32, 704-713. doi:10.1016/j.neuroimage.2006.04.189

[47] Takahashi, T., Suzuki, M., Kawasaki, Y., Kurokawa, K., Hagino, H., Yamashita, I., Zhou, S.Y., Nohara, S., Nakamura, K., Seto, H. and Kurachi, M. (2002) Volumetric magnetic resonance imaging study of the anterior cingulate gyrus in schizotypal disorder. European Archives of Psychiatry and Clinical Neurosciences, 252, 268-277. doi:10.1007/s00406-002-0392-3

[48] Kopp, B., Mattler, U., Goertz, R. and Rist, F. (1996) N2, P3 and the lateralized readiness potential in a NoGo task involving selective response priming. Electroencephalography and Clinical Neurophysiology, 99, 19-27. doi:10.1016/0921-884X(96)95617-9 